\title{
The VirB5 protein localizes to the T-pilus tips in Agrobacterium tumefaciens
}

Correspondence

Christian Baron

baronc@mcmaster.ca

Received 7 June 2007

Revised 22 July 2007

Accepted 6 August 2007

\author{
Khaled A. Aly and Christian Baron
}

\author{
McMaster University, Department of Biology and Antimicrobial Research Centre, 1280 Main \\ Street West, Hamilton, ON L8S 4K1, Canada
}

\begin{abstract}
The Agrobacterium tumefaciens VirB/D4 type IV secretion system (T4SS) mediates the transfer of single-stranded DNA and protein virulence factors into plant cells, and also determines the assembly of the T-pilus, which is believed to play a role in host recognition. The T-pilus is composed of the major component VirB2 and the minor component VirB5. Using immuno-electron microscopy we detected the major component VirB2 along the entire length of detached T-pili, but not on cell-bound T-pili or on the cell surface. In contrast, the minor T-pilus component VirB5 was detected on the tips of cell-bound T-pili as well as on the ends of detached T-pili and on the cell surface. To gain further insights into the role of VirB5 we introduced changes at its $\mathrm{C}$ terminus. C-terminal deletions of up to four amino acids and alanine replacements did not abolish T-pilus formation and incorporation of the VirB5 variants at the tip, although they did impact the length of T-pili. Also, these changes differentially affected the ability of the T4SS to transfer DNA into plant and bacterial recipients, suggesting differential effects on host-cell specificity. The data presented here suggest that VirB5 localizes at the T-pilus tip, and provide novel insights into its role during the type IV secretion process.
\end{abstract}

\section{INTRODUCTION}

Many Gram-negative bacteria assemble extracellular highmolecular-mass filamentous structures such as pili on their surface, which are typically composed of one protein or a small set of proteins. Binding is the principal function in the case of adhesive pili, such as $\mathrm{P}$ and type- 1 pili, which mediate the attachment of bacteria to host tissues to initiate colonization (Kau et al., 2005; Sauer et al., 2004). In contrast, type IV secretion system (T4SS)-determined surface structures are involved in a complex cascade of events that lead to the inter-bacterial or trans-kingdom delivery of effectors, mostly proteinaceous virulence factors, but also DNA molecules (Baron, 2005; Christie et al., 2005; Yeo \& Waksman, 2004). T4SSs are essential for the virulence of many pathogens, such as Agrobacterium tumefaciens (gene transfer and tumour formation on plants), Bartonella henselae (cat scratch disease), Bordetella pertussis (whooping cough), Helicobacter pylori (gastritis and stomach cancer) and Legionella pneumophila (Legionnaires' disease) (Backert \& Meyer, 2006; Baron, 2005; Christie et al., 2005; McCullen \& Binns, 2006; Schröder \& Dehio, 2005).

VirB2-like proteins are the major pilus components of the A. tumefaciens, plasmid RP4 and F-determined T4SSs, and

Abbreviations: AS, acetosyringone; EM, electron microscopy; immunoEM, immuno-electron microscopy; T4SS, type IV secretion system; TDNA, transferred DNA; TEM, transmission electron microscopy.

Supplementary data are available with the online version of this paper. homologues exist in most other systems (Fullner et al., 1996; Kalkum et al., 2002; Lai \& Kado, 1998). VirB2 proteins are small and hydrophobic, and after cleavage of their signal peptides, those from A. tumefaciens and RP4 are processed to cyclic peptides (Eisenbrandt et al., 1999; Kalkum et al., 2002). Interaction partners of A. tumefaciens VirB2 pilin have been identified in an Arabidopsis thaliana cDNA expression library, implying that the major T-pilus protein may directly interact with host-cell components (Hwang \& Gelvin, 2004).

Whereas the presence of VirB2-like major pilin proteins is well established, knowledge of minor pilus components and their function(s) is relatively limited. Minor components may play a role in pilus elongation, but in a similar manner to tip components of adhesive pili, they may also initiate contact with host cells. There is some indirect evidence for the existence of an adhesin at the F-pilus tip (Anthony et al., 1994), but so far, minor pilus components have not been identified in this system. VirB5-like proteins have been shown to be minor components of pili determined by the A. tumefaciens, IncN plasmid pKM101 and IncP plasmid pJP4 T4SSs (Schmidt-Eisenlohr et al., 1999a, b, 2001), and VirB5 is essential for the incorporation of the major component VirB2 into T-pili (Lai et al., 2000; Schmidt-Eisenlohr et al., 1999a). The X-ray structure of the pKM101 VirB5 homologue TraC has been solved, and structure-function analysis has identified residues important for DNA transfer and binding to pilus-specific bacteriophages (Yeo et al., 2003). These results constitute 
indirect evidence for a role of externally localized $\mathrm{TraC}$ in cell adhesion, a possibility that was originally proposed based on the discovery that $\operatorname{traC}$ deletion variants of pKM101 can be complemented extracellularly (Winans \& Walker, 1985). However, earlier work has not directly assessed the localization of VirB5-like proteins in T4SSdetermined pili and the possibility that they may play a role in host-cell contact formation.

Apart from VirB5, there is no firm evidence for other minor T-pilus components. The lipoprotein VirB7 cofractionates with extracellular high-molecular-mass structures such as T-pili after shearing from A. tumefaciens, indicating that it may be a part of this structure (Sagulenko et al., 2001), although this fractionation is not dependent on pilus formation. These results suggest that upon lipidation, VirB7 may become a T-pilus assembly factor, and its cofractionation with VirB2 and VirB5 after detergent extraction accords with this view (Yuan et al., 2005). The fact that its fractionation does not depend on Tpilus formation indicates that VirB7 may associate with high-molecular-mass membrane blebs sheared from the cell, but that it is not likely to be an integral pilus component (Sagulenko et al., 2001). Another externally localized VirB component is $\operatorname{VirB} 1^{\star}$, the C-terminal processing product of VirB1 (Baron et al., 1997). This fragment of VirB1 is secreted into the supernatant, and it is possible that it contributes to host-cell recognition (Llosa et al., 2000), but so far, it has not been found to associate with T-pili.

A pilus assembly sequence has recently been proposed, and according to this model the inner membrane-bound NTPase VirB4 stabilizes VirB8, which then mediates interactions between VirB2 and VirB5 followed by T-pilus incorporation (Yuan et al., 2005). VirB3 is also stabilized by VirB4, it fractionates with VirB2 and VirB5 after extraction with the mild detergent dodecyl- $\beta$-D-maltoside, and it interacts with VirB5; these results indicate that it may also play a role in T-pilus assembly (Jones et al., 1994; Shamaei-Tousi et al., 2004). A key feature of this model is the formation of a VirB2-VirB5 T-pilus preassembly subcomplex (Krall et al., 2002; Yuan et al., 2005), but further understanding of this process requires clarification of the localization of VirB2 and VirB5 in the assembled structure. VirB5 may function as an outer-membrane usher, similar to PapC of the P-pilus system, and the outer membrane would be its final localization in this case (Sauer et al., 2004). Alternatively, VirB5 may initiate T-pilus formation after its export into the periplasm; it may become incorporated at the T-pilus tips and also localize inside the pilus. In order to decide between these different models of T-pilus assembly, it is crucial to localize T-pilus components in the assembled structure.

To this end, we have analysed the localization of VirB2 and VirB5 in the T-pilus by immuno-electron microscopy (immuno-EM). Using a specific antiserum we detected VirB2 in T-pili isolated by shearing and ultracentrifugation but not in cell-bound T-pili, indicating that these structures may undergo conformational changes after removal from the cells. We have also determined the functionality of the T4SS and the length of T-pili in the wild-type strain C58 and in strains expressing VirB5 variants with changes at the $\mathrm{C}$ terminus. These analyses showed that the VirB5 C terminus impacts T-pilus incorporation of the protein and T-pilus length, and that it differentially affects DNA transfer to bacterial versus plant cells. The experiments reported here suggest that the minor component VirB5 localizes at the T-pilus tip, whereas VirB2 localizes along its entire length. Combined with the results of our analysis of the effects of C-terminal variants on host-cell specificity, these data suggest that tiplocalized VirB5 impacts host-cell recognition.

\section{METHODS}

Cultivation of bacteria, and strain and plasmid construction. Escherichia coli JM109 for cloning experiments and A. tumefaciens for virulence gene induction were cultivated on $\mathrm{AB}$ minimal medium with acetosyringone (AS) and $0.5 \mathrm{mM}$ IPTG for induction of plasmidencoded genes as described previously (Höppner et al., 2004; Yuan et al., 2005). DNA manipulations followed standard procedures (Maniatis et al., 1982). The A. tumefaciens virB5 gene in pTrcB5 (Schmidt-Eisenlohr et al., 1999a) was amplified by PCR with oligonucleotides to introduce C-terminal deletions or changes into the gene product (Supplementary Table S3) and cloned into expression vector $p \operatorname{Trc} 200$. The sequences of PCR-amplified genes were confirmed by DNA sequencing. The virD4 deletion strain CB2004 was generated from the wild-type C58 by PCR-amplification of the virD4 gene and deletion of an internal fragment, followed by integration into the chromosome by established methods (Berger \& Christie, 1994).

Analysis of T4SS functions: T-pilus isolation, conjugation and virulence assay. Assays for T4SS functionality (T-pilus isolation, conjugation and virulence assays) were performed as previously described (Höppner et al., 2004; Yuan et al., 2005).

SDS-PAGE and Western blotting. SDS-PAGE was conducted according to Laemmli (1970) for proteins $>20 \mathrm{kDa}$, or Schägger \& von Jagow (1987) for proteins $<20 \mathrm{kDa}$. Western blotting was performed following standard protocols with VirB protein-specific antisera, as described previously (Harlow \& Lane, 1988; Yuan et al., 2005).

T-pilus purification by gel filtration. T-pili from different $A$. tumefaciens strains were isolated by shearing of the cells and ultracentrifugation, according to established procedures (Höppner et al., 2004; Yuan et al., 2005). For further purification, the piluscontaining sediments obtained after ultracentrifugation were resuspended in $100 \mu \mathrm{l} 50 \mathrm{mM}$ sodium potassium phosphate (NaKP) buffer, $\mathrm{pH} 5.5$, and applied to a Superdex 200 column (GE Healthcare), followed by separation with a flow rate of $0.5 \mathrm{ml} \mathrm{min}{ }^{-1}$. The fractions eluting from the column were concentrated by the addition of three volumes of acetone and incubation on ice for $4 \mathrm{~h}$. The samples were then subjected to ultracentrifugation for $2 \mathrm{~h}$ at $270000 \mathrm{~g}$ for precipitation of the pili, followed by resuspension in $40 \mu \mathrm{l} \mathrm{NaKP}$ buffer. Thirty microlitres of these samples were mixed with equal volumes of Laemmli sample buffer followed by SDS-PAGE and Western blot analysis. The remaining samples were loaded on electron microscopy (EM) grids for further visualization of purified T-pili. 
Transmission electron microscopy (TEM). A. tumefaciens strains were grown under virulence-induction conditions on solid $\mathrm{AB}$ agar, as previously reported (Yuan et al., 2005), and collected with liquid $\mathrm{AB}$ minimal medium supplemented with $\mathrm{AS}$, and the cell density was adjusted to $\mathrm{OD}_{600} 0.25$. Next, $15 \mu \mathrm{l}$ was applied to UV-sterilized 200 or 300 mesh carbon-coated Formvar nickel EM grids, and the cells were cultivated for $10-12 \mathrm{~h}$ at $20{ }^{\circ} \mathrm{C}$ in a humid chamber to permit T-pilus elongation and to minimize their breakage. The cells were then stained with $1 \%$ phosphotungstic acid in $0.01 \%$ glucose $(\mathrm{pH} 6)$ for $15-45 \mathrm{~s}$, followed by EM examination.

For T-pilus length analysis, we measured 88,77 and 67 cell-bound Tpili of strains C58, CB1005 p TrcB5 $\Delta 3$ and $\mathrm{CB} 1005$ p $\operatorname{TrcB} 5 \Delta 4$, respectively. The length of the T-pili was measured directly from the micrographs and scaled using a standard EM correction grid. Similarly, we also measured the length of 300 cells from pole to pole. Micrographs from three independent virulence-induction experiments were analysed. For quantification of T-pilus formation, experiments were conducted as described above, followed by more intense staining with $2 \%$ phosphotungstic acid, $0.01 \%$ glucose, $\mathrm{pH} 6$, for $15-20 \mathrm{~s}$ to efficiently label the pili. Depending on the experiment, T-pili from 60-400 cells from three independent virulence-induction experiments of each strain were counted, and 10 cells per visual field were analysed.

For immuno-EM, strains were cultivated under virulence geneinducing conditions on EM grids as above, and the grids were then fixed for $1 \mathrm{~h}$ with $2 \%$ formaldehyde, $0.5 \%$ glutaraldehyde in $50 \mathrm{mM}$ sodium cacodylate buffer, pH 5.5, as described by Jin et al. (2001). After fixation, the grids were subjected to immunogold labelling, largely as described by Quintero et al. (1998). The grids were blocked with $5 \%$ skimmed milk in TBST $(20 \mathrm{mM}$ Tris/ $\mathrm{HCl}, 137 \mathrm{mM} \mathrm{NaCl}, 0.1 \%$ Tween-20, $\mathrm{pH} 8$ ) for $20 \mathrm{~min}$, followed by incubation on a drop of either $1: 250$ diluted anti-VirB5 or $1: 250$ anti-VirB2 antiserum in $5 \%$ skimmed milk and TBST for $45 \mathrm{~min}$ at room temperature. The grids were then washed three times for $5 \mathrm{~min}$, incubated with the secondary antibodies for $45 \mathrm{~min}$ at room temperature (1:10 diluted anti-rabbit $10 \mathrm{~nm}$ gold conjugate; Sigma-Aldrich) in TBST with $5 \%$ skimmed milk, $0.1 \%$ BSA and $5 \%$ fetal bovine serum. Finally, the grids were washed and negative-stained with $1 \%$ phosphotungstic acid in $0.01 \%$ glucose ( $\mathrm{pH}$ 6) for $15 \mathrm{~s}$ prior to examination. For quantification of the percentage of VirB5-labelled tips on T-pilus carrying C58, CB1005 pTrcB5 $\Delta 3$ and CB1005 pTrcB5 $\Delta 4$ cells, we analysed 300 T-piluscarrying cells from three independent experiments.

The electron microscopic images were recorded with a JEOL JEM$1200 \mathrm{EX}$ or a JEOL 1200EX II transmission electron microscope.

\section{RESULTS}

\section{Deletion of C-terminal amino acids leads to gradual loss of T-pilus incorporation of VirB5}

A. tumefaciens assembles three different types of surfaceexposed appendages, which can be easily differentiated based on their different diameters (flagella, $15 \mathrm{~nm}$; T-pili, $10 \mathrm{~nm}$; common pili, $3 \mathrm{~nm}$ ) (Fig. 1a) (Eisenbrandt et al., 1999; Lai et al., 2000). The approach reported here was inspired by the analysis of the X-ray structure of $\mathrm{TraC}$, which had suggested that the C-terminal 15-20 amino acids are likely exposed on the surface of the protein and may therefore be available for protein-protein interactions (Yeo et al., 2003). To assess the functional importance of this region, we deleted the gene to express VirB5 variants truncated by blocks of five amino acids (deletion of five to (a)

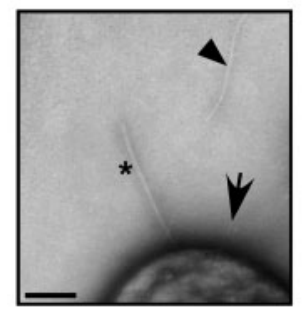

(b)

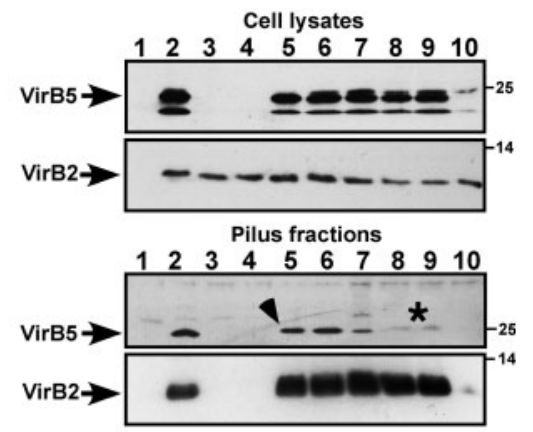

(c)

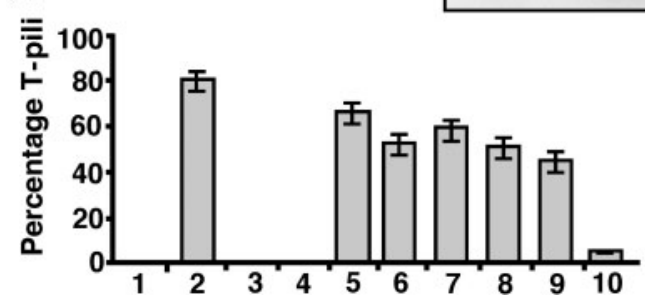

Fig. 1. Effects of C-terminal deletions of VirB5 on T-pilus formation. (a) Representative micrograph of the different surface appendages assembled by $A$. tumefaciens. The asterisk points to a flagellum (diameter $15 \mathrm{~nm}$ ), the arrow points to a common pilus (diameter $3 \mathrm{~nm}$ ) and the arrowhead points to a T-pilus (diameter $10 \mathrm{~nm}$ ). (b) Samples from subcellular fractions were separated by SDS-PAGE, followed by Western blot analysis with VirB2- and VirB5-specific antisera. The analysis is shown of wild-type C58, CB1005 (AvirB5) and CB1005 complemented with pTrc200 expressing VirB5 and its variants grown on $A B$ minimal medium under virulence-inducing (+AS, lanes 2-10) and non-inducing (-AS, lane 1) conditions. IPTG (0.5 mM) was added to induce transcription of the pTrc200-encoded virB5 genes. Lanes: 1, C58 -AS; 2, C58 +AS; 3, CB1005; 4, CB1005 carrying pTrc200; 5, pTrcB5; $6, p \operatorname{TrcB} 5 \Delta 1 ; 7, p \operatorname{TrcB} 5 \Delta 2 ; 8, p \operatorname{TrcB} 5 \Delta 3 ; 9, \mathrm{p} \operatorname{TrcB} 5 \Delta 4$; $10, p \operatorname{TrcB} 5 \Delta 5$. The arrowhead indicates pilus incorporation of VirB5 in complemented CB1005 and the asterisk indicates the detection of low amounts of $\operatorname{VirB} 5 \Delta 3$ and $\operatorname{VirB} 5 \Delta 4$ in pilus fractions (lanes 8 and 9). Numbers on the right indicate molecular masses of reference proteins. (c) Quantification of results of the TEM analysis of pilus formation (percentage detection of one or more T-pili per cell) [numbering of bars as in (b); representative images shown as Supplementary Fig. S1]. Forty different fields (10 cells each) from three different inductions were counted for each strain and the SD is shown.

30 amino acids) in the A. tumefaciens strain C58 virB5 gene-deletion mutant CB1005 (Schmidt-Eisenlohr et al., 1999a). The genes were cloned into the broad-host-range plasmid p Trc200, followed by analysis of the localization of the gene products. Analysis of VirB5 in cell lysates showed that all the deletion variants accumulated in strongly reduced amounts, and pili containing VirB2 and VirB5 were not formed (not shown). We next created deletions in virB5 to direct synthesis of VirB5 variants truncated in oneamino-acid steps from the $\mathrm{C}$ terminus. Analysis of the lysates (from CB1005 p TrcB5 $\Delta 1, \mathrm{p} \operatorname{TrcB} 5 \Delta 2$, etc.) showed 
that deletion of up to four amino acids did not affect the stability of VirB5 in the cell, but we observed a gradual loss of T-pilus incorporation. Whereas deletion of one amino acid (VirB5 $\Delta 1$ ) did not notably affect the levels of VirB5 in T-pili, successive deletions led to reduced amounts, although the proteins were still detected in T-pilus fractions (Fig. 1b). In contrast, the incorporation of VirB2 into T-pili was not notably affected by truncations of VirB5 of up to four amino acids, which further supported the notion that T-pili were still formed (Fig. 1b).

T-pilus formation was next visualized and quantified by TEM of virulence gene-induced agrobacteria (Supplementary Fig. S1). One or more T-pili were detected on the surface of $79 \%$ of the cells in virulence-induced strain C58 and of $63 \%$ in the case of CB1005 pTrcB5 (Fig. 1c). Deletions at the $\mathrm{C}$ terminus of up to four amino acids led only to minor reductions of the amounts of surfaceexposed T-pili, but the number was strongly reduced in CB1005 pTrcB5 $\Delta 5$, as this VirB5 variant was not stable. Similarly, no T-pili were observed in CB1005 producing VirB5 variants with further deletions that were not stable in the cell (not shown).

The amounts of VirB5 $\Delta 3$ and VirB5 $\Delta 4$ variants associated with T-pili were significantly reduced (Fig. 1b), and it was therefore necessary to conduct additional purification steps to assess their association with T-pili. To this end, T-pili isolated from the cells by shearing and ultracentrifugation as above were resuspended in a small volume of buffer and separated by gel filtration over a Superdex 200 sizeexclusion column. The eluted fractions were concentrated by acetone precipitation, followed by SDS-PAGE and Western blotting to determine the elution volume of VirB2, and of VirB5 and its truncated variants. This analysis showed that VirB5 wild-type as well as $\operatorname{VirB5} \Delta 3$ and VirB5 $\Delta 4$ co-eluted with VirB2 in the high-molecularmass range, as in our previous work (Schmidt-Eisenlohr et al., 1999a), and similar to the analysis above, the amounts of C-terminally truncated variants were reduced as compared to the wild-type (Fig. 2a). Electron microscopic analysis of these fractions confirmed the presence of T-pili (Fig. 2b). Thus, in spite of the reduced amounts of C-terminally truncated VirB5 variants, our data suggest that they associate with T-pili in a similar manner to wildtype VirB5.

\section{The VirB5 C terminus impacts T-pilus length}

When we monitored T-pilus production in CB1005 producing C-terminal deletion variants of VirB5 we noticed that the pilus length differed from that of the wild-type in all cases. CB1005 p $\operatorname{TrcB} 5 \Delta 1-\Delta 3$ produced notably shorter $\mathrm{T}$ pili, whereas those of CB1005 p TrcB5 $\Delta 4$ appeared on average to be longer than those of the wildtype. To quantify this phenomenon we conducted virulence-gene induction of the wild-type C58, CB1005 pTrcB5 $\Delta 3$ and CB1005 pTrcB5 $\Delta 4$ as above, and measured the length of cell-bound T-pili after negative staining and

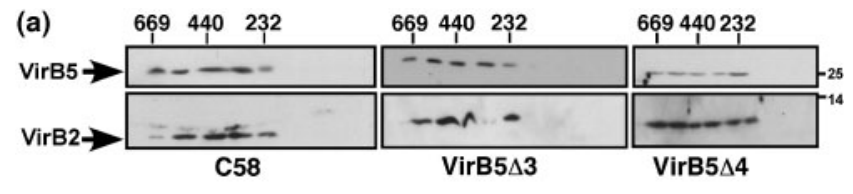

(b)

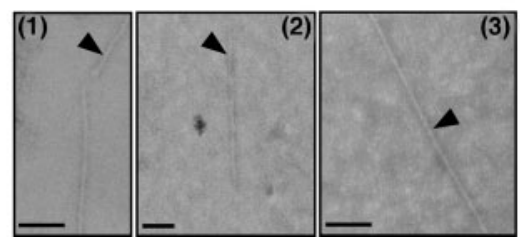

Fig. 2. Gel-filtration analysis of T-pili containing VirB5 and Cterminal deletion variants. Surface structures isolated by shearing and high-speed centrifugation from agrobacteria grown under virulence gene-inducing conditions were further separated by gelfiltration chromatography. Column fractions were subjected to SDS-PAGE followed by Western blotting with specific antisera for VirB2 or VirB5. (a) VirB2 and VirB5 detected in the highmolecular-mass fractions eluted from the column after separation of samples from strains C58, CB1005 pTrcB5 $\Delta 3$ and CB1005 pTrcB5 $\Delta 4$. Numbers on the top indicate molecular masses of reference proteins thyroglobulin $(669 \mathrm{kDa})$, ferritin $(440 \mathrm{kDa})$ and catalase $(232 \mathrm{kDa})$. (b) TEM micrographs of T-pili (indicated by arrowheads) from strains C58 (1), CB1005 pTrcB5 $\Delta 3$ (2) and $\mathrm{CB} 1005 \mathrm{pTrcB5} \Delta 4$ (3) after gel-filtration chromatography. Bars, $100 \mathrm{~nm}$.

TEM. The mean T-pilus length in strain C58 was $1390 \mathrm{~nm}$, and we occasionally observed pili with a length of up to $4500 \mathrm{~nm}$ (Fig. 3). As a comparison, the mean length of virulence gene-induced wild-type C58 cells was $1480 \mathrm{~nm}$ (maximum length, $2250 \mathrm{~nm}$; minimum length, $1020 \mathrm{~nm}$ ). In contrast to the results obtained for the wild-type, the mean T-pilus lengths observed for cells of CB1005 pTrcB5 $\Delta 3$ and CB1005 pTrcB5 $\Delta 4$ were 455 and $1750 \mathrm{~nm}$, respectively. Whereas these results confirmed our initial observations, we also noticed that the maximum lengths of pili assembled on strains CB1005 p $\operatorname{TrcB} 5 \Delta 3$ and CB1005 pTrcB5 $\Delta 4$ were 1500 and $3,500 \mathrm{~nm}$, respectively. These results show that in spite of the increased mean length of T-pili formed on CB1005 p TrcB5 $\Delta 4$, they do not reach the maximum length of those on the wild-type, indicating a more complex effect of the C-terminal deletion than variation in length. Thus, C-terminal deletions of VirB5 do have differential effects on T-pilus length as measured by $\mathrm{EM}$, and we next assessed the functionality of these structures during the type IV secretion process.

\section{Truncations of the $\mathbf{C}$ terminus differentially affect the functionality of VirB5 in gene transfer}

The A. tumefaciens T4SS transfers substrates to different hosts (Lacroix et al., 2006), and we here used bacteria and plants as recipients to assess the functionality of C-terminal VirB5 deletion variants. First, we tested transferred DNA (T-DNA) transfer in a plant infection assay leading to 

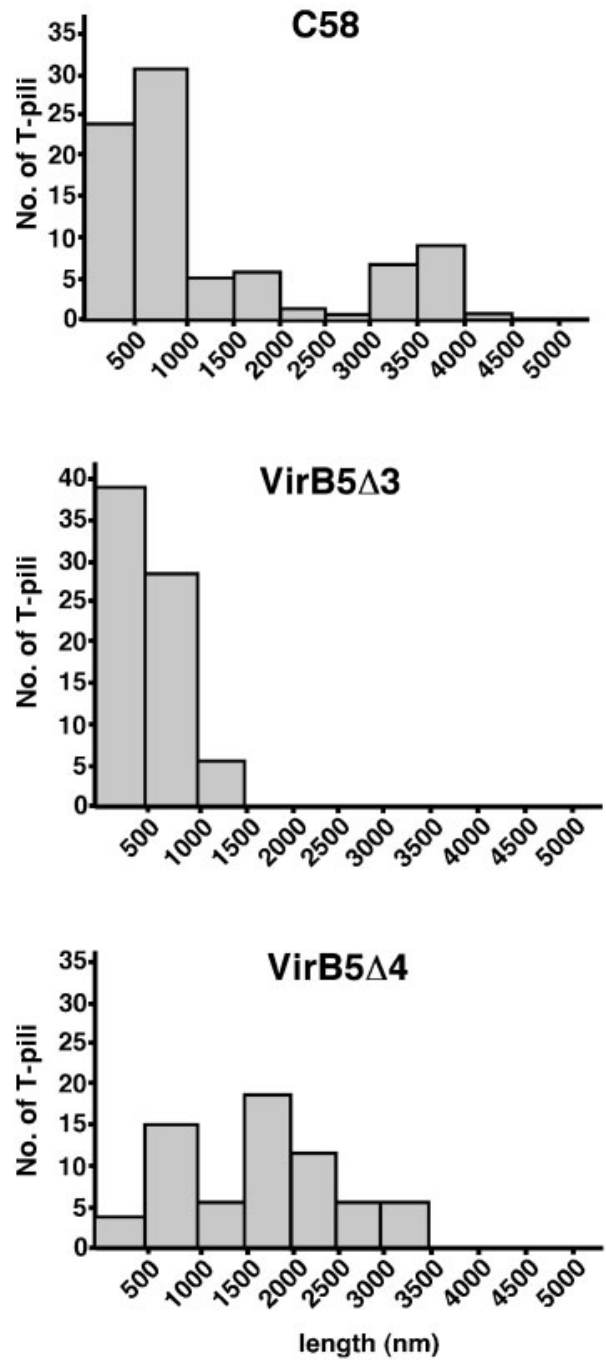

Fig. 3. C-terminal deletions of VirB5 impact the length of T-pili. $A$. tumefaciens strains C58, CB1005 pTrcB5 $\Delta 3$ and CB1005 $\mathrm{p} \operatorname{TrcB5} \Delta 4$ were grown under virulence-inducing conditions, followed by negative straining and TEM to visualize the surfaceexposed T-pili. The lengths of 88,77 and 67 cell-bound T-pili on strains C58, CB1005 pTrcB5 B5 $\Delta 3$ and CB1005 pTrcB5 B5 $\Delta 4$, respectively, were measured using a magnification-correction grid. T-pili on cells from three independent virulence-induction experiments were analysed.

tumour formation on wounded Kalanchoë diagremontiana leaves. Second, as a more quantitative assay, we exploited the ability of the T4SS to direct conjugative transfer of the IncQ plasmid pLS1 between agrobacteria (Stahl et al., 1998; Ward et al., 1991). The results of both assays were comparable in most cases, but we noted a few intriguing differences. The virB5 deletion strain $\mathrm{CB} 1005$ did not incite tumours after wounding and infection of $K$. diagremontiana leaves; this defect was complemented by expression of wild-type VirB5, but the CB1005 strains expressing Cterminal deletion variants (VirB5 $\Delta 1-\Delta 5)$ were all avirulent
(Supplementary Fig. S2A). Using the conjugation assay, we found that transfer of pLS1 from the virB5 deletion strain was reduced to $1.5-10 \%$ in different independent series of experiments (Tables 1 and 2). Complementation with VirB5 increased pLS1 transfer to $50-75 \%$ of the wild-type. Expression of VirB5 $\Delta 1-\Delta 3$ and VirB5 $\Delta 5$ did not complement, but in contrast to the results of the plant infection experiments, production of VirB5 $\Delta 4$ fully complemented pLS1 transfer to A. tumefaciens (Table 1). These results showed that truncation of the $\mathrm{C}$ terminus differentially affected gene transfer into the recipients $K$. diagremontiana and Agrobacterium.

\section{C-terminal amino acid changes differentially affect the stability and functionality of VirB5}

As the C-terminal amino acids apparently have a major impact on the functionality of VirB5 we next mutagenized the gene to change the sequences of the last two amino acids from AVP to AAA, AVA and AAP. The variants were produced in $\mathrm{CB} 1005$, and analysis of their subcellular localization showed that VirB5AAA and VirB5AAP were detected in the cells and T-pili in levels similar to those of the wild-type (Fig. 4a). In contrast, VirB5AVA was unstable and was not detected in the cells or the T-pili, which may be due to recognition of this sequence by a protease in the bacterial cytoplasm or periplasm. Analysis by TEM confirmed that T-pili were formed on CB1005 pTrcB5AAA and $\mathrm{CB} 1005$ pTrcB5AAP, but not on the strain carrying pTrcB5AVA (Fig. 4b). Following the analysis of their subcellular localization, we next analysed the functionality of C-terminal VirB5 alanine variants in gene transfer, and they all proved to be avirulent in plant infection assays (Supplementary Fig. S2b). In contrast, in the pLS1 plasmid transfer assay, VirB5AAP was partly functional (69\% of the wild-type), whereas VirB5AAA and VirB5AVA did not complement (Table 2). Thus, similar to the results obtained with C-terminal deletion variants, subtle changes of the C-terminal amino acid sequences differentially affected gene transfer into the two recipients used here. As the localization of VirB2 and VirB5 is key to understand their role in T-pilus function, we addressed this question next.

\section{Immuno-EM detects VirB2 in detached but not in cell-bound T-pili}

The A. tumefaciens virulence genes were induced as above and the cells fixed on EM grids, followed by detection with VirB2-specific primary and $10 \mathrm{~nm}$ gold-labelled secondary antisera. Analysis by TEM revealed that gold grains did not label VirB2 on cell-bound intact T-pili, and we also did not detect any label on the cell surface (Fig. 5a, b). However, we did notice gold grain label on pilus-like structures that were detached from the cells (Fig. 5b). These structures often had an irregular shape unlike the straight and elongated T-pili, and may represent pili that were partly degraded during cultivation or the EM preparation 
Table 1. Analysis of pLS1 transfer from A. tumefaciens donors A348, virB5 deletion strain PC1005 and complemented derivatives into A. tumefaciens UIA143 pTiA6

The SD was based on three repetitions; TC, transconjugants.

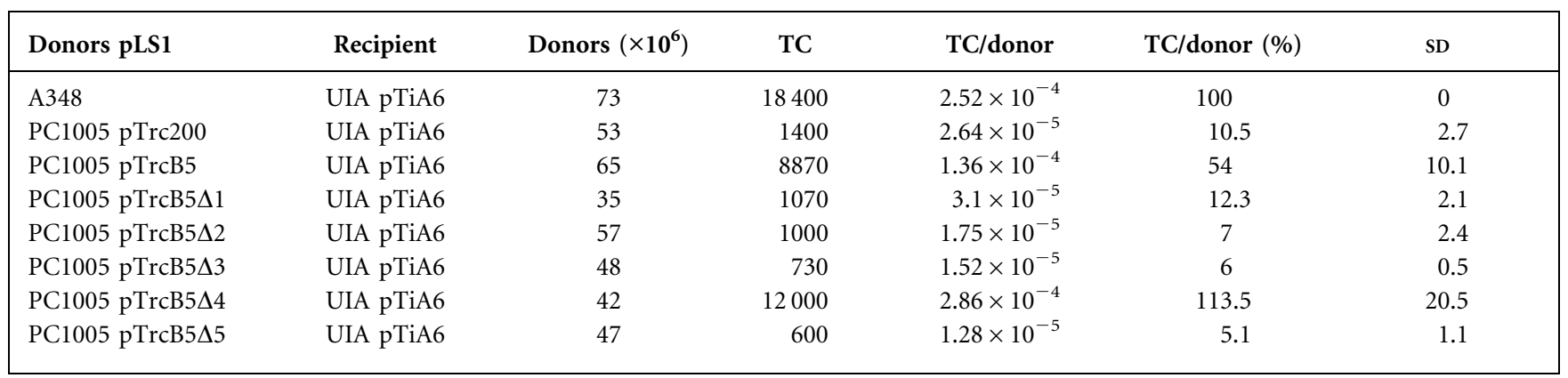

procedure. This observation opened the possibility that the epitope recognized by the antibodies may be accessible in detached T-pili. To assess this possibility, we analysed Tpili isolated from the cells by shearing and ultracentrifugation. In isolated T-pili, we detected gold grain label along the entire length (Fig. 5c, d) of $96 \%$ of the T-pili isolated from strain C58, and at slightly lower levels on T-pili from CB1005 expressing the VirB5 $\Delta 3$ and VirB5 $\Delta 4$ variants (Supplementary Table S1). Gold grains were exclusively detected on detached (sheared) T-pili and not on cellbound pili or flagella. The results were similar in the wildtype strain C58 (Fig. 5a-d) and in the virD4 deletion variant CB2004 (Fig. 5e). These results indicate that the accessibility of the epitope recognized by the VirB2-specific antiserum differs between cell-bound and detached T-pili, and that the coupling of substrates mediated by VirD4 does not impact this phenomenon.

\section{Immuno-EM detects VirB5 on the cell surface and on the T-pilus tips}

We next performed immuno-EM analysis with a VirB5specific antiserum and $10 \mathrm{~nm}$ gold-labelled secondary antiserum, and detected the label in three different locations: on the cell surface, on the tips of T-pili, and on the ends of T-pili isolated by shearing and ultracentrifugation. Quantification of our observations revealed that an average of nine gold grains per cell was detected on the surface, showing that in contrast to VirB2, cell-bound VirB5 was readily accessible to antibodies (Fig. 6a). Gold labels on the T-pilus tips were detected on $26 \%$ of the Tpilus-carrying wild-type cells (Fig. $6 \mathrm{~b}, \mathrm{c}$ ) and they were also detected at a similar percentage on the ends of T-pili isolated by shearing and ultracentrifugation (Fig. 6f, Supplementary Table S1), but not along the length of Tpili. Similar observations were made in the virD4 deletion strain CB2004 (Fig. 6g), showing that coupling of the substrate to the T4SS does not impact the localization of VirB5. Controls with non-induced or virulence-induced cells incubated without primary or secondary antibodies showed that the detection of VirB5 was specific (Supplementary Fig. S3A-D). Also, using specific antisera and immuno-EM analysis as above, we did not detect VirB1 or VirB7 on the cell surface and on the T-pili (results not shown). In the case of these two proteins, partial exterior localization has previously been demonstrated (Baron et al., 1997; Sagulenko et al., 2001), and we concluded either that they are not present on the surface and in the T-pili or that the epitopes were not accessible to the antisera under the conditions used here.

In the experiments described above, VirB5 variants with shortened $\mathrm{C}$ termini proved to be particularly interesting, as these changes impacted pilus length as well as gene transfer to different hosts. To assess whether VirB5 also localized on the tips of these pili, we analysed CB1005

Table 2. Analysis of pLS1 transfer from A. tumefaciens donors A348, virB5 deletion strain PC1005 and complemented derivatives into A. tumefaciens UIA143 pTiA6

The SD was based on three repetitions; TC, transconjugants.

\begin{tabular}{|lccrrrr|}
\hline Donors pLS1 & Recipient & Donors $\left(\times \mathbf{1 0}^{\mathbf{6}}\right)$ & TC & TC/donor & TC/donor $(\mathbf{\%})$ \\
\hline A348 & UIA pTiA6 & 48 & 18900 & $3.93 \times 10^{-4}$ & 100 & SD \\
PC1005 pTrc200 & UIA pTiA6 & 52 & 300 & $5.7 \times 10^{-6}$ & 1.5 \\
PC1005 pTrcB5 & UIA pTiA6 & 57 & 16800 & $2.95 \times 10^{-4}$ & 75.1 & 0.7 \\
PC1005 pTrcB5AAA & UIA pTiA6 & 47 & 200 & $4.2 \times 10^{-6}$ & 1.1 & 0.3 \\
PC1005 pTrcB5AVA & UIA pTiA6 & 52 & 100 & $1.9 \times 10^{-6}$ & 0.5 \\
PC1005 pTrcB5AAP & UIA pTiA6 & 56 & 15300 & $2.72 \times 10^{-4}$ & 69.2 & 0.04 \\
& & & & & 11.1 \\
\hline
\end{tabular}


(a)

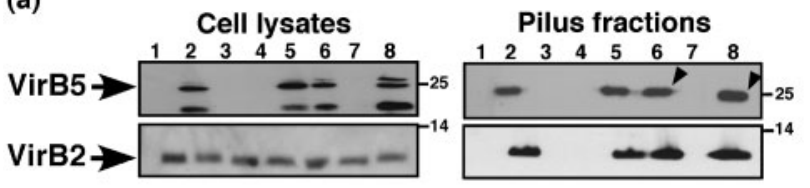

(b)

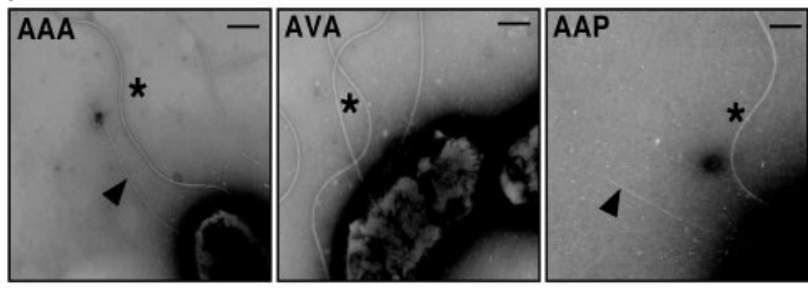

Fig. 4. Effects of C-terminal alanine changes of VirB5 on T-pilus formation. The analysis is shown of wild-type C58, CB1005 and CB1005 complemented with pTrc200 expressing VirB5 and its variants cultivated under virulence-inducing ( $+A S)$ and noninducing (-AS) conditions. IPTG $(0.5 \mathrm{mM})$ was added to induce the transcription of pTrc200-encoded virB5 genes. (a) Samples from subcellular fractions were separated by SDS-PAGE, followed by Western blot analysis with VirB2- and VirB5-specific antisera. Lanes: 1, C58 -AS; 2, C58 +AS; 3, CB1005; 4, CB1005 pTrc200; 5, pTrcB5; 6, pTrcB5AAA; 7, pTrcB5AVA; 8, pTrcB5AAP. Arrowheads indicate detection of VirB5 variants in pilus fractions. Numbers on the right indicate molecular masses of reference proteins. (b) TEM analysis of C58 and CB1005 producing $\mathrm{C}$-terminal alanine variants of VirB5 (C-terminal amino acids given: VirB5AAA, VirB5AVA and VirB5AAP). Asterisks show flagella and arrowheads point to T-pili. Bars, $100 \mathrm{~nm}$.

$\mathrm{p} \operatorname{TrcB} 5 \Delta 3$ and $\mathrm{CB} 1005 \mathrm{p} \operatorname{TrcB} 5 \Delta 4$, and gold grains were detected on the pilus tips in both cases (Fig. 6d, e). When these data were quantified it became apparent that compared to the wild-type ( $26 \%$ ), a higher percentage of T-pilus tips was labelled by gold grains in the case of CB1005 pTrcB5 $\Delta 3(55 \%)$, and a lower percentage in the case of $\mathrm{CB} 1005 \mathrm{p} \operatorname{TrcB} 5 \Delta 4 \quad(7 \%)$; we made similar observations with isolated T-pili (Supplementary Table S2). Thus, in spite of the reduced levels detected by Western blotting in detached T-pili, these results show that both VirB5 $\Delta 3$ and VirB5 $\Delta 4$ localize to the tips of T-pili.

\section{DISCUSSION}

The work presented here provides direct evidence for the localization of the major component VirB2 and of the minor component VirB5 in T-pili. We first analysed the localization of VirB2 in T-pili using intact cells and immuno-EM, but the VirB2-specific antiserum did not target gold-labelled secondary antibodies to cell-bound pili or to the cell surface. However, we occasionally noticed the labelling of curled structures by multiple gold grains, and these had the approximate diameter of T-pili, though not their mostly straight appearance. These curled structures

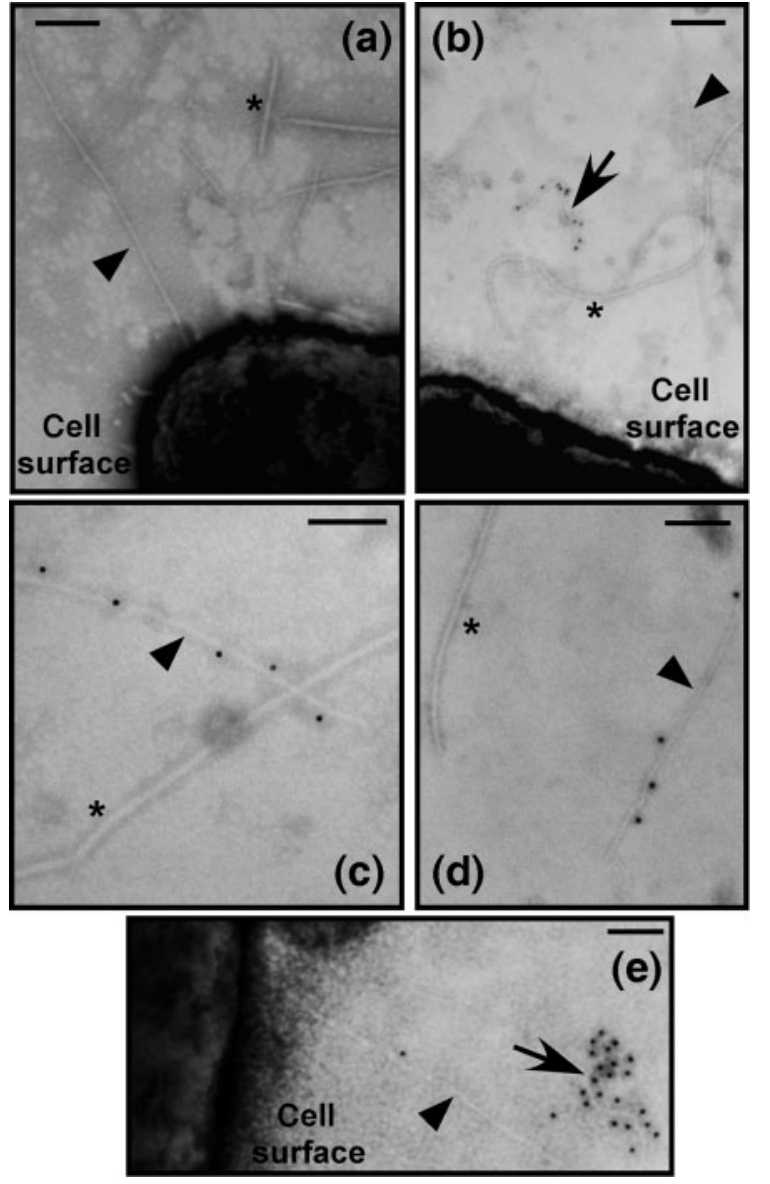

Fig. 5. Immuno-EM analysis of cell-bound and detached T-pili with VirB2-specific antiserum. The wild-type strain C58 was cultivated under virulence-inducing conditions followed by immuno-EM analysis of cell-bound T-pili $(a, b)$. Alternatively, Tpili were detached from the cells by shearing, concentrated by ultracentrifugation and analysed by immuno-EM (c, d). (e) Analysis of virulence-induced virD4 deletion strain CB2004. Detection with VirB2-specific primary and $10 \mathrm{~nm}$ gold-coupled secondary antisera is shown; bars, $100 \mathrm{~nm}$. Arrowheads show T-pili, asterisks point to flagella, and arrows indicate the detection of the VirB2 antigen in detached T-pilus-like structures in (b) and (e).

are reminiscent of T-pili treated with detergents, acids or bases (Lai \& Kado, 2002). They may correspond to T-pili that were detached from the cell and partly degraded during growth under virulence gene-inducing conditions or by the EM fixation procedure. These observations indicated that the VirB2 epitope may not be accessible in cell-bound pili, and to assess this possibility, we isolated Tpili from the cells that were fragmented by shearing and then sedimented by ultracentrifugation. Analysis of isolated T-pili by immuno-EM with VirB2-specific antiserum targeted gold grain-labelled secondary antibodies to almost $100 \%$ of the isolated T-pili, and we typically observed several grains per pilus. These results suggest that the epitope recognized by the VirB2-specific antiserum, which 


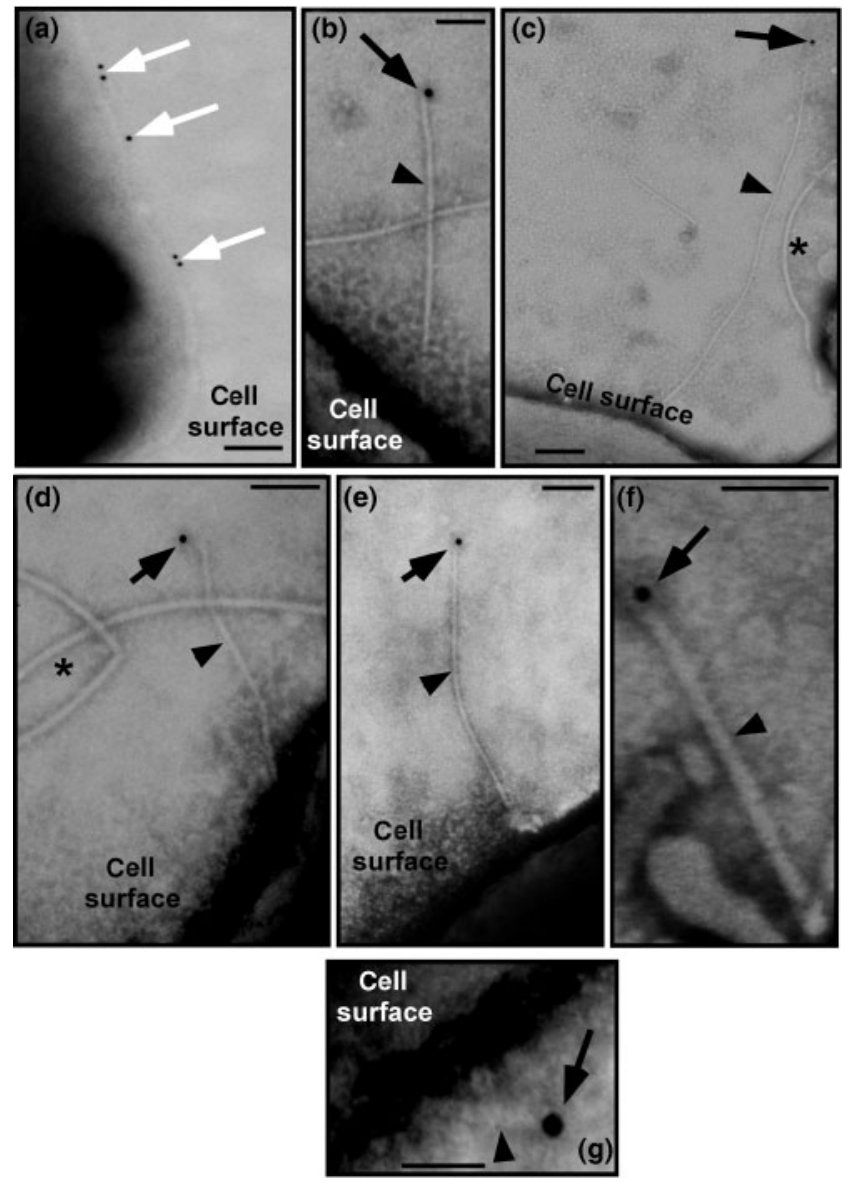

Fig. 6. Immuno-EM analysis of cell-bound and isolated T-pili with VirB5-specific antiserum. Cells were cultivated under virulenceinducing conditions, followed by immuno-EM analysis (a-e) or Tpilus isolation by shearing and ultracentrifugation followed by immuno-EM analysis (f). Analysis of the wild-type strain C58 (a-c, f), CB1005 pTrcB5 $\Delta 3$ (d), CB1005 pTrcB5 $\Delta 4$ (e) and the virD4 deletion strain CB2004 (g) is shown. Detection with VirB5specific primary and $10 \mathrm{~nm}$ gold-coupled secondary antisera is shown; bars, $100 \mathrm{~nm}$. Arrowheads show T-pili, asterisks point to flagella, and black arrows show the detection of gold grains on Tpilus tips (b-f). (a) An Agrobacterium cell; white arrows point to gold grains that indicate the localization of VirB5 on the cell surface.

was generated against a 15 amino acid peptide immediately adjacent to the processing and cyclization sequence (Carle et al., 2006), may be hidden in intact T-pili. A conformational change upon detachment from the cells, possibly triggered by experimental manipulation, makes it accessible. It is not clear whether this conformational change has any biological significance, but it is tempting to speculate that it reflects a change of the pilus conformation upon host-cell binding and/or during substrate translocation. To address this question we analysed the localization of VirB2 and VirB5 in the virD4 deletion strain CB2004, which does not couple the translocated T-complex to the T4SS. The results were identical to those of the wild-type, showing that substrate coupling does not have an effect on T-pilus conformation that is detectable with the methods used here. High-resolution structural studies, e.g. by cryoelectron microscopy and image reconstruction, will be required in future to investigate the nature of this conformational change (Craig et al., 2006). We will also conduct immuno-EM experiments that include host cells (plant or bacterial) in order to assess whether host recognition by T-pili leads to conformational changes that unmask the epitope recognized by the VirB2-specific antiserum.

We next analysed the localization of the minor T-pilus component and detected VirB5 on the tips of T-pili and on the cell surface. VirB5 was detected on the tips of $26 \%$ of the pili in the wild-type strain C58, and one possible explanation for this incomplete labelling is that surfaceexposed structures such as T-pili may break off during the preparation for EM. This is in accord with a model that implies that VirB5 initiates T-pilus assembly in the cell envelope and remains at the tip upon pilus elongation. According to this model, the major pilus subunit may be delivered to the pilus base by the VirB2-VirB5 complex that we described previously (Yuan et al., 2005), followed by the degradation of VirB5 and successive addition of VirB2 subunits at the base. As a consequence, VirB5 may not be present inside pili and was therefore not detected on the ends of broken pili. This model would explain why VirB5 was detected on only $26 \%$ of the cell-bound pili and why a similar degree of labelling $(34 \%)$ was also observed on detached pili. The analysis of VirB5 variants with Cterminal deletions lent further support to this model.

Analysis of CB1005 expressing VirB5 $\Delta 3$ resulted in a higher number of tip-labelled pili on cells ( $55 \%)$, and a similar amount of label was detected on detached T-pili (56\%). This elevated level of labelling in comparison to the wildtype may be due to the fact that these pili are shorter and may therefore not break off and fragment as easily. A similar reasoning may explain the fact that only $7 \%$ of the tips on cells of CB1005 expressing VirB5 $\Delta 4$ were labelled (11\% in the case of detached T-pili). These pili are on average longer than those from C58, and they may break off more easily and further break into pieces, which may explain the lower level of tip labelling. In spite of having longer pili on average, the maximum length of pili on CB1005 expressing VirB5 $\Delta 4$ was shorter than in the wildtype, which is also in accord with a structure that may break off more easily. Nevertheless, the overall structure of these pili appears to be similar to that of the wild-type, and the detached pili from CB1005 expressing VirB5 $\Delta 3$ and VirB5 $\Delta 4$ were labelled with the VirB2-specific antiserum along their entire length, like those from the wild-type. There is little precedent for the length regulation of surface appendages, but that of type III secretion systemdetermined needles from Yersinia enterocolitica is determined by a domain of $\mathrm{YscP}$ in a molecular ruler-like fashion (Journet et al., 2003; Mota et al., 2005). The 
discovery that minor changes of VirB5 lead to notable variations of pilus length opens up a fascinating area of research for future work.

The above-mentioned model does not exclude alternative mechanisms of T-pilus assembly, e.g. the incorporation of VirB2 subunits at the tip after transfer via the pilus lumen, as in the case of the subunits of bacterial flagella. Also, VirB5 may be present inside the T-pilus, where it cannot be readily detected by antisera, but the facts that it is only a minor pilus component (Schmidt-Eisenlohr et al., 1999a) and that we did not detect higher levels of labelling on detached in comparison to cell-bound T-pili argue against this possibility. An alternative explanation for the localization of VirB5 at the tips may be that it can be sheared from the cell surface in the form of membrane blebs, which may bind to the tips of broken T-pili. Although this possibility cannot be ruled out, there is currently no published evidence for the shearing of VirB5-containing membrane blebs from A. tumefaciens, and we have not observed such structures in the course of our electron microscopic studies. In any case, the detection of VirB5 at the pilus tip as well as on the cell surface argues in favour of a sequence of interactions that lead to T-pilus assembly following initiation by a cell-bound form of VirB5 in the periplasm and/or on the surface. As discussed below, the effects of C-terminal deletions and changes on the efficacy of DNA transfer to different hosts are also consistent with the notion that the tip is one of the natural localizations of VirB5.

In addition to providing interesting clues for future research on T-pilus assembly, the analysis of C-terminal VirB5 variants provided novel insights into the host specificity of the DNA translocation process. None of the C-terminal VirB5 variants analysed here (C-terminal deletions as well as alanine replacements) complemented the defect of the CB1005 mutant in plant-infection assays. The variants with deletions of up to four amino acids and with two of the three alanine changes were stable in the cell, indicating that the avirulence of these strains was not due to degradation of the protein but that the $\mathrm{C}$ terminus of VirB5 is important for T-DNA transfer to plants. The finding that relatively minor changes at the $\mathrm{C}$ terminus abolished DNA transfer to plants was unexpected, and there are three possible explanations. First, many of our assumptions are based on the X-ray structure of TraC, which suggests a surface-exposed $\mathrm{C}$ terminus. VirB5 and TraC have only limited sequence similarity ( $14 \%$ identical and $33 \%$ similar amino acids; see alignment in Supplementary Fig. S4), but this portion of VirB5 may localize inside the protein, and even minor changes may have caused drastic conformational changes. We do not consider this possibility very likely, as most of the noncomplementing variants were stable in the cell, which would not be expected if their overall structure were changed. Also, a variant with a C-terminal extension of eight amino acids (StrepII tag) was fully functional (not shown) and this is also in accord with an accessible C terminus. Second, VirB5 may interact via its C terminus with translocated substrates so that changes directly block the translocation of DNA and/or of proteins via the T4SS. This is indeed an interesting possibility that warrants further study, but so far, there is no evidence for an interaction between VirB5 and the translocated substrates. The third explanation is that VirB5 may be involved in binding to plant cells, and this hypothesis is in accord with the proposed role of the VirB5 homologue TraC in phage adhesion and plasmid transfer (Yeo et al., 2003). This notion is supported by our data that show that two of the VirB5 variants that did not complement T-DNA transfer to plants (VirB5 $\Delta 4$ and VirB5AAP) did complement the defect of a virB5 deletion strain in transfer of the IncQ plasmid pLS1 to recipient agrobacteria. The fact that VirB5 $\Delta 4$ and VirB5AAP complemented the virB5 defect in this assay indicates that the T-pili engaged fully in DNAand protein-transfer processes and that recognition of the recipient by VirB5 may play a role during the transfer process. This view is consistent with the results of our EM work, which showed that VirB5 localized at the pilus tip, which is suggestive of a role in host-cell recognition. Nevertheless, in spite of the evidence provided here and in previous work (Yeo et al., 2003), a function of VirB5 as an adhesin is still hypothetical. Future work will directly test this possibility, e.g. by screening for interaction partners with the yeast two-hybrid system or by using biochemical approaches to isolate VirB5-binding proteins from plants and $\mathrm{TraC} /$ VirB5-binding proteins from bacteria.

\section{ACKNOWLEDGEMENTS}

We are indebted to Patricia Zambryski (University of California, Berkeley) for continued support and discussions, Marcia Reid (McMaster University) for help with EM and John Lott (McMaster University) for help with pilus-length measurements. This work was supported by grants from the Natural Sciences and Engineering Research Council of Canada (NSERC; grant 262104), the Canada Foundation for Innovation (CFI) and the Ontario Innovation Trust (OIT) to C. B.

\section{REFERENCES}

Anthony, K. G., Sherbourne, C., Sherburne, R. \& Frost, L. S. (1994). The role of the pilus in recipient cell recognition during bacterial conjugation mediated by F-like plasmids. Mol Microbiol 13, 939-953.

Backert, S. \& Meyer, T. F. (2006). Type IV secretion systems and their effectors in bacterial pathogenesis. Curr Opin Microbiol 9, 207-217.

Baron, C. (2005). From bioremediation to biowarfare: on the impact and mechanism of type IV secretion systems. FEMS Microbiol Lett 253, 163-170.

Baron, C., Llosa, M., Zhou, S. \& Zambryski, P. C. (1997). C-terminal processing and cellular localization of VirB1, a component of the Tcomplex transfer machinery of Agrobacterium tumefaciens. J Bacteriol 179, 1203-1210.

Berger, B. R. \& Christie, P. J. (1994). Genetic complementation analysis of the Agrobacterium tumefaciens virB operon: virB2 through virB11 are essential virulence genes. J Bacteriol 176, 3646-3660. 
Carle, A., Höppner, C., Aly, K. A., Yuan, Q., den Dulk-Ras, A., Vergunst, A., O'Callaghan, D. \& Baron, C. (2006). The Brucella suis type IV secretion system assembles in the cell envelope of the heterologous host Agrobacterium tumefaciens and increases IncQ plasmid pLS1 recipient competence. Infect Immun 74, 108-117.

Christie, P. J., Atmakuri, K., Krishnamoorthy, V., Jakubowski, S. \& Cascales, E. (2005). Biogenesis, architecture, and function of bacterial type IV secretion systems. Annu Rev Microbiol 59, 451-485.

Craig, L., Volkmann, N., Arvai, A. S., Pique, M. E., Yeager, M., Egelman, E. H. \& Tainer, J. A. (2006). Type IV pilus structure by cryoelectron microscopy and crystallography: implications for pilus assembly and functions. Mol Cell 23, 651-662.

Eisenbrandt, R., Kalkum, M., Lai, E. M., Lurz, R., Kado, C. I. \& Lanka, E. (1999). Conjugative pili of IncP plasmids, and the Ti plasmid T pilus are composed of cyclic subunits. J Biol Chem 274, 22548-22555.

Fullner, K. J., Lara, J. L. \& Nester, E. W. (1996). Pilus assembly by Agrobacterium T-DNA transfer genes. Science 273, 1107-1109.

Harlow, E. \& Lane, D. (1988). Antibodies: a Laboratory Manual. Cold Spring Harbor, NY: Cold Spring Harbor Laboratory.

Höppner, C., Liu, Z., Domke, N., Binns, A. N. \& Baron, C. (2004). VirB1 orthologs from Brucella suis and pKM101 complement defects of the lytic transglycosylase required for efficient type IV secretion from Agrobacterium tumefaciens. J Bacteriol 186, 1415-1422.

Hwang, H. H. \& Gelvin, S. B. (2004). Plant proteins that interact with VirB2, the Agrobacterium tumefaciens pilin protein, mediate plant transformation. Plant Cell 16, 3148-3167.

Jin, Q., Hu, W., Brown, I., McGhee, G., Hart, P., Jones, A. L. \& He, S. Y. (2001). Visualization of secreted Hrp and Avr proteins along the Hrp pilus during type III secretion in Erwinia amylovora and Pseudomonas syringae. Mol Microbiol 40, 1129-1139.

Jones, A. L., Shirasu, K. \& Kado, C. I. (1994). The product of the virB4 gene of Agrobacterium tumefaciens promotes accumulation of VirB3 protein. J Bacteriol 176, 5255-5261.

Journet, L., Agrain, C., Broz, P. \& Cornelis, G. R. (2003). The needle length of bacterial injectisomes is determined by a molecular ruler. Science 302, 1757-1760.

Kalkum, M., Eisenbrandt, R., Lurz, R. \& Lanka, E. (2002). Tying rings for sex. Trends Microbiol 10, 382-387.

Kau, A. L., Hunstad, D. A. \& Hultgren, S. J. (2005). Interaction of uropathogenic Escherichia coli with host uroepithelium. Curr Opin Microbiol 8, 54-59.

Krall, L., Wiedemann, U., Unsin, G., Weiss, S., Domke, N. \& Baron, C. (2002). Detergent extraction identifies different VirB protein subassemblies of the type IV secretion machinery in the membranes of Agrobacterium tumefaciens. Proc Natl Acad Sci U S A 99, 1140511410 .

Lacroix, B., Tzfira, T., Vainstein, A. \& Citovsky, V. (2006). A case of promiscuity: Agrobacterium's endless hunt for new partners. Trends Genet 22, 29-37.

Laemmli, U. K. (1970). Cleavage of structural proteins during the assembly of the head of bacteriophage T4. Nature 227, 680-685.

Lai, E.-M. \& Kado, C. I. (1998). Processed VirB2 is the major subunit of the promiscuous pilus of Agrobacterium tumefaciens. J Bacteriol 180, 2711-2717.

Lai, E. M. \& Kado, C. I. (2002). The Agrobacterium tumefaciens T pilus composed of cyclic $\mathrm{T}$ pilin is highly resilient to extreme environments. FEMS Microbiol Lett 210, 111-114.

Lai, E. M., Chesnokova, O., Banta, L. M. \& Kado, C. I. (2000). Genetic and environmental factors affecting T-pilin export and T-pilus biogenesis in relation to flagellation of Agrobacterium tumefaciens. J Bacteriol 182, 3705-3716.
Llosa, M., Zupan, J., Baron, C. \& Zambryski, P. C. (2000). The N- and C-terminal portions of the Agrobacterium VirB1 protein independently enhance tumorgenesis. J Bacteriol 182, 3437-3445.

Maniatis, T. A., Fritsch, E. F. \& Sambrook, J. (1982). Molecular Cloning: a Laboratory Manual. Cold Spring Harbor, NY: Cold Spring Harbor Laboratory.

McCullen, C. A. \& Binns, A. N. (2006). Agrobacterium tumefaciens and plant cell interactions and activities required for interkingdom macromolecular transfer. Annu Rev Cell Dev Biol 22, 101-127.

Mota, L. J., Journet, L., Sorg, l., Agrain, C. \& Cornelis, G. R. (2005). Bacterial injectisomes: needle length does matter. Science 307, 1278.

Quintero, E. J., Busch, K. \& Weiner, R. M. (1998). Spatial and temporal deposition of adhesive extracellular polysaccharide capsule and fimbriae by Hyphomonas strain MHS-3. Appl Environ Microbiol 64, 1246-1255.

Sagulenko, V., Sagulenko, E., Jakubowski, S., Spudich, E. \& Christie, P. J. (2001). VirB7 lipoprotein is exocellular and associates with the Agrobacterium tumefaciens T-pilus. J Bacteriol 183, 3642-3651.

Sauer, F. G., Remaut, H., Hultgren, S. J. \& Waksman, G. (2004). Fiber assembly by the chaperone-usher pathway. Biochim Biophys Acta 1694, 259-267.

Schägger, H. \& von Jagow, G. (1987). Tricine-sodium dodecyl sulfate-polyacrylamide gel electrophoresis for the separation of proteins in the range of 1 to $100 \mathrm{kDa}$. Anal Biochem 166, 368-379.

Schmidt-Eisenlohr, H., Domke, N., Angerer, C., Wanner, G., Zambryski, P. C. \& Baron, C. (1999a). Vir proteins stabilize VirB5 and mediate its association with the $\mathrm{T}$ pilus of Agrobacterium tumefaciens. J Bacteriol 181, 7485-7492.

Schmidt-Eisenlohr, H., Domke, N. \& Baron, C. (1999b). TraC of IncN plasmid pKM101 associates with membranes and extracellular high molecular weight structures in Escherichia coli. J Bacteriol 181, 5563-5571.

Schmidt-Eisenlohr, H., Rittig, M., Preithner, S. \& Baron, C. (2001). Biomonitoring of pJP4-carrying Pseudomonas chlororaphis with Trb protein-specific antisera. Environ Microbiol 3, 720-730.

Schröder, G. \& Dehio, C. (2005). Virulence-associated type IV secretion systems of Bartonella. Trends Microbiol 13, 336-342.

Shamaei-Tousi, A., Cahill, R. \& Frankel, G. (2004). Interaction between protein subunits of the type IV secretion system of Bartonella henselae. J Bacteriol 186, 4796-4801.

Stahl, L. E., Jacobs, A. \& Binns, A. N. (1998). The conjugal intermediate of plasmid RSF1010 inhibits Agrobacterium tumefaciens virulence and VirB-dependent export of VirE2. J Bacteriol 180, 3933-3939.

Ward, J. E., Dale, E. M. \& Binns, A. N. (1991). Activity of the Agrobacterium T-DNA transfer machinery is affected by $\operatorname{vir} B$ gene products. Proc Natl Acad Sci U S A 88, 9350-9354.

Winans, S. C. \& Walker, G. C. (1985). Conjugal transfer system of the $\mathrm{N}$ incompatibility plasmid pKM101. J Bacteriol 161, 402-410.

Yeo, H.-J. \& Waksman, G. (2004). Unveiling molecular scaffolds of the type IV secretion system. J Bacteriol 186, 1919-1926.

Yeo, H.-J., Yuan, Q., Beck, M. R., Baron, C. \& Waksman, G. (2003). Structural and functional characterization of the VirB5 protein from the type IV secretion system encoded by the conjugative plasmid pKM101. Proc Natl Acad Sci U S A 100, 15947-15962.

Yuan, O., Carle, A., Gao, C., Sivanesan, D., Aly, K., Höppner, C., Krall, L., Domke, N. \& Baron, C. (2005). Identification of the VirB4VirB8-VirB5-VirB2 pilus assembly sequence of type IV secretion systems. J Biol Chem 280, 26349-26359.

Edited by: M. S. Ullrich 